\title{
MODELING AND KINEMATICS SIMULATION OF PLANE TEN-BAR MECHANISM OF WARP KNITTING MACHINE BASED ON SIMCAPE/MULTIBODY
}

\author{
Zhonghua Yan \\ Changde Textile Machinery Co. Ltd, Hunan, Changde, 415001, P.R.China. \\ *Corresponding Author Email: 13873670621@163.com
}

This is an open access article distributed under the Creative Commons Attribution License, which permits unrestricted use, distribution, and reproduction in any medium, provided the original work is properly cited

\section{ARTICLE DETAILS}

\section{Article History:}

Received 12 January 2018 Accepted 12 February 2018 Available online 1 June 2018

\section{ABSTRACT}

A kinematics simulation model was established for the driven-latch needle of warp knitting machine, and the simulation was implemented by using SimMultibody. Thus, the simulation curves of displacement, velocity and acceleration for a certain key point of the mechanism were obtained. The modeling analysis is implemented for a certain mechanical system. The results show that this method is convenient, efficient and powerful. It provides a theoretical foundation and a better analytical approach of simulation for the design and analysis of complex multilinkage mechanisms.

\section{KEYWORDS}

Matlab, SimMultibody, Driven-latch needle of warp knitting machine, Plane ten-bar mechanism, Kinematics, Simulation.

\section{INTRODUCTION}

Conveyor Knitting as one main branch of textile is a new textile industry rapidly growing accompanied by the progress of traditional processing technology and modern information technology. Warp knitting machine is one of high-end knitting machines. Warp knitting industry has driven the technological innovation and progress of new materials, machinery manufacturing, agriculture, water conservancy, health care and other areas. Knitting machine is currently developing towards high speed, high efficiency, high precision, intelligence, diversification, differentiation, good stability and high reliability [1]. Most of the current high-speed warp knitting machines use compound grooved drum needle, and the highest speed can be up to $3000 \mathrm{r} / \mathrm{min}$. The design technology of high-speed warp knitting machine mainly involves mechanism dynamics analysis, precision manufacturing technology, new material application, wear resistance improvement, etc. This technology has been very mature at abroad. E.g., the HKS2-3E Tricot groove-needle type of speed warp knitting machine of German Karl Mayer Company (E36, double comb short stroke) has the maximum speed of up to $4000 \mathrm{r} / \mathrm{min}$. The RSE4-1-type speed Raschel machine (E32) can reach $2600 \mathrm{rpm} \mathrm{r} / \mathrm{min}$ [2].

Modern high-speed warp knitting machine generally uses crank and connecting rod mechanism to replace cam mechanism. Crank and connecting rod mechanism is lower-pair connection component, having performances of small contact stress and good dynamic performance. Moreover, it avoids the mechanical vibration caused by cam follower and cam gap. Thus, it has been widely used [3]. Mechanism motion analysis is the basis of mechanism design, motion evaluation and motion control. There are generally two forms of mechanism motion analyses: forward analysis and backward analysis. Forward analysis is solving the motion parameters of follower in mechanism with the known motion of driving member. Backward analysis is solving the motion parameters of other components in mechanism with the known motion of end component. The motion analysis of mechanism is performed usually by the graphical and analytical methods. The applications of graphical method in practical engineering have significant limitations due to its shortcomings of large mapping workload and poor accuracy. Analytical method can be achieved through programing, SimMechanics simulation as well as SimMultibody simulation based on Matlab software [4-6]. SimMultibody uses graphical modeling approach and automatically selects integration algorithm.
Moreover, it can monitor the change in equation characteristics and automatically transform integral algorithm in the integral process to obtain the best results. Thus, SimMultibody does not need to derive complex mathematical model and can pay more attention to problem, which is high in simulation efficiency and easy to use. Therefore, the graphical modeling approach of SimMultibody simulation software can be used to conveniently build warp-knitting-machine simulation model for dynamic demonstration, kinematics and dynamics analyses. This work performed the kinematics modeling and simulation of plane ten-bar mechanism of transmission latch needle in warp knitting machine based on SimMultibody.

\section{SIMMULTIBODY SOFTWARE}

The Simcape family of products consists of six products, namely, Simcape ${ }^{\mathrm{TM}}$, SimDriveline $\mathrm{T}^{\mathrm{TM}}$, SimElectronics ${ }^{\mathrm{TM}}$, SimFluids ${ }^{\mathrm{TM}}$, SimMultibody $^{\mathrm{TM}}$, SimPower Systems ${ }^{\mathrm{TM}}$. Simcape enables you to build models using components in the Foundation libraries, create custom components, simulate models that include multi-domain schematics and analyze simulation results. You can combine any set of the Simcape add-on products with the Simcape platform to model multi-domain physical systems. The add-on products include more advanced blocks and analysis methods.

SimMultibody ${ }^{\mathrm{TM}}$ (formerly SimMechanics ${ }^{\mathrm{TM}}$ ) provides a multibody simulation environment for 3D mechanical systems, such as robots, vehicle suspensions, construction equipment, and aircraft landing gear. You can model multibody systems using blocks representing bodies, joints, constraints, force elements, and sensors. SimMultibody formulates and solves the equations of motion for the complete mechanical system. You can import complete CAD assemblies, including all masses, inertias, joints, constraints, and 3D geometry, into your model. An automatically generated 3D animation lets you visualize the system dynamics. SimMultibody helps you develop control systems and test system-level performance. You can parameterize your models using MATLAB $®$ variables and expressions, and design control systems for your multibody system in Simulink®. You can integrate hydraulic, electrical, pneumatic, and other physical systems into your model using components from the $\mathrm{Sim}^{\mathrm{TM}}$ family of products. To deploy your models to other simulation environments, including hardware-in-the-loop (HIL) systems, 
SimMultibody supports C-code generation. SimMultibody provides much of the core technology and capabilities necessary for modeling and simulating physical systems in all domains.

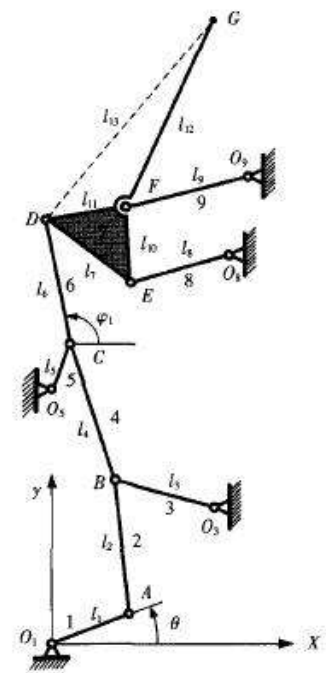

Figure 1: Ten-bar mechanism of driven-latch-needle of warp loom

\section{SIMULATION OF LATCH NEEDLE MECHANISM}

Transmission latch-needle mechanism is an important part of warp knitting machine. Figure 1 shows the ten-bar mechanism sketch of transmission latch-needle plane of warp knitting machine [5]. Given mechanism dimensions were as follows:

$$
\begin{aligned}
& I_{1}=30, I_{2}=150, I_{3}=45, I_{4}=60, I_{5}=20, \\
& I_{6}=252, I_{7}=120.42, I_{8}=80, I_{9}=100 \\
& I_{10}=90, I_{11}=80, I_{12}=229.89, I_{13}=274.32, \\
& 0_{1 x}=0_{1 y}=0,0_{3 x}=90,0_{3 y}=130,0_{5 x}=0 \\
& 0_{5 y}=170,0_{8 x}=156,0_{8 y}=340,0_{9_{x}}=17 \\
& 0_{9_{y}}=430
\end{aligned}
$$

The angular velocity of driving crank is $\dot{\theta}=20 \mathrm{rad} / \mathrm{s}$, and the position, velocity and acceleration of key point $\mathrm{G}$ were solved.

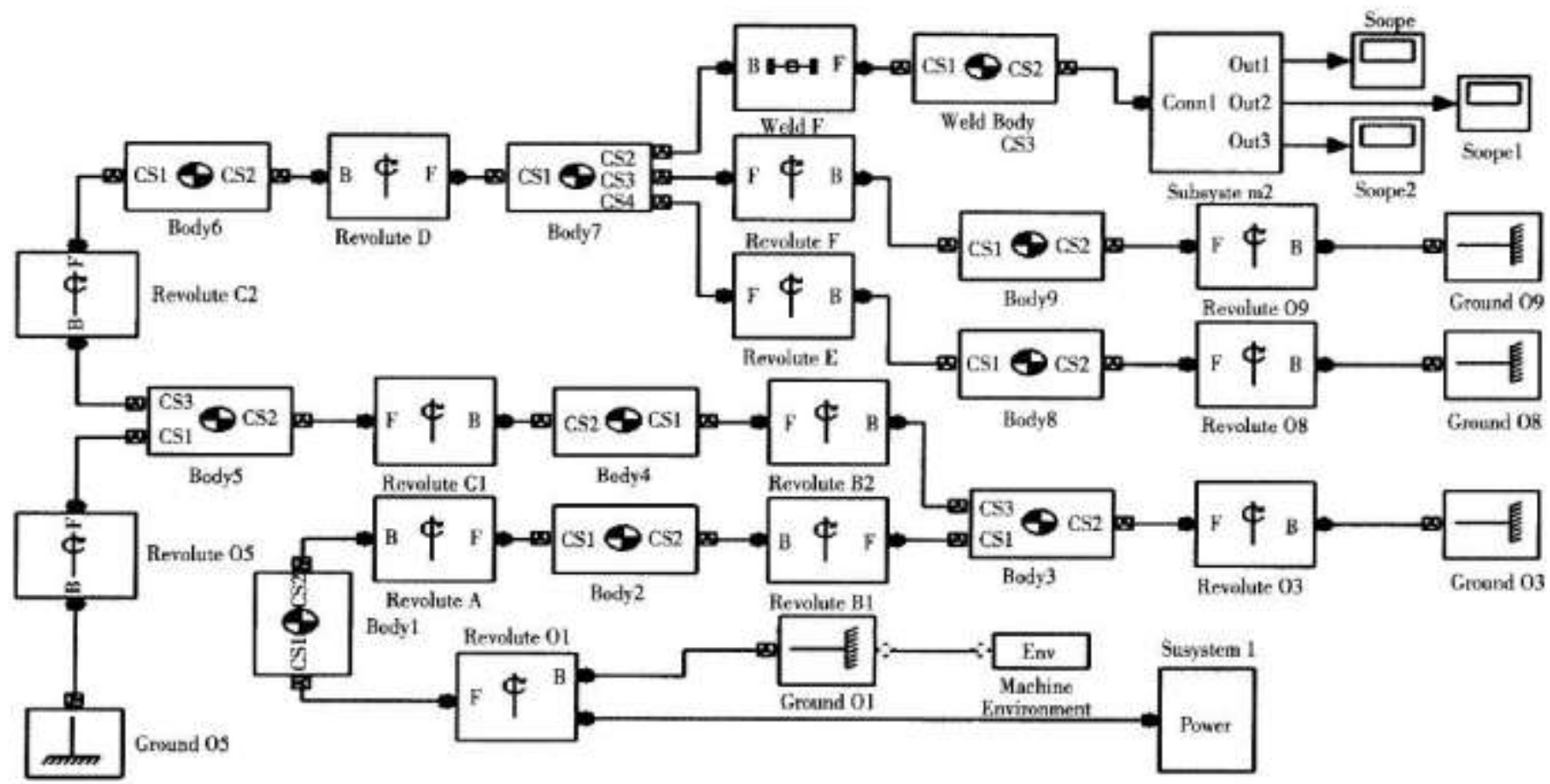

Figure 2: Multibody simulation model of mechanism
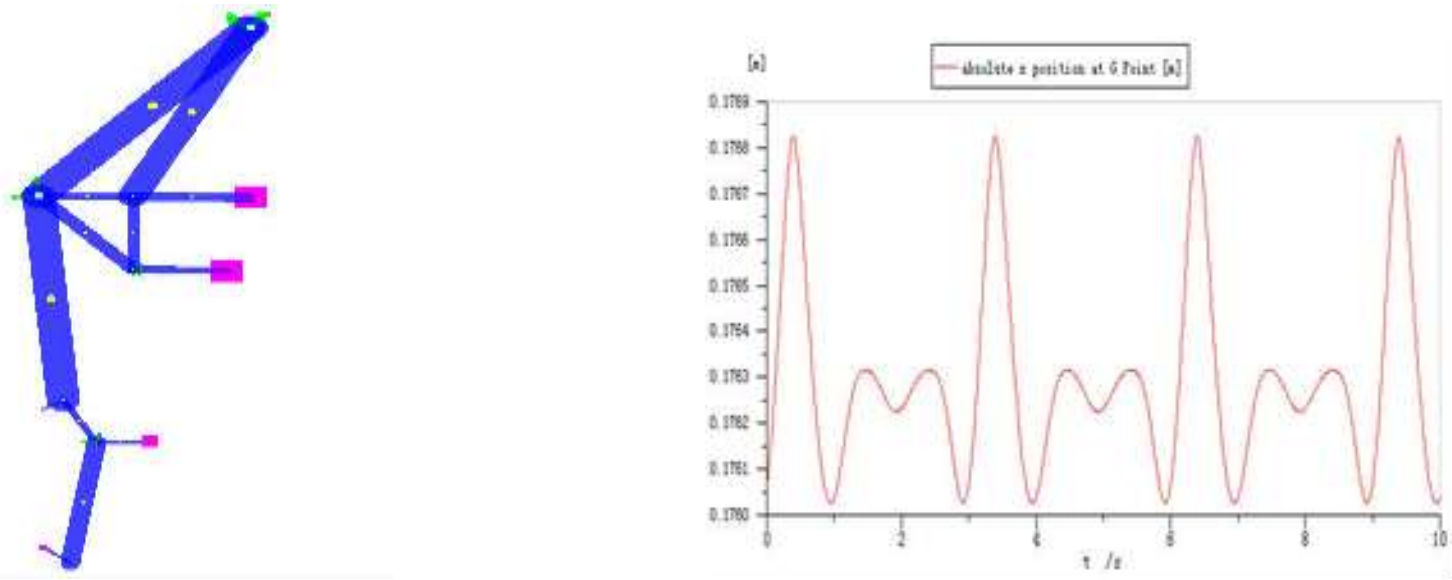

Figure 3: Animation display of mechanism

Figure 4: Absolute $\mathrm{x}$ position at $\mathrm{G}$ point 




Figure 5: Absolute y position at G point

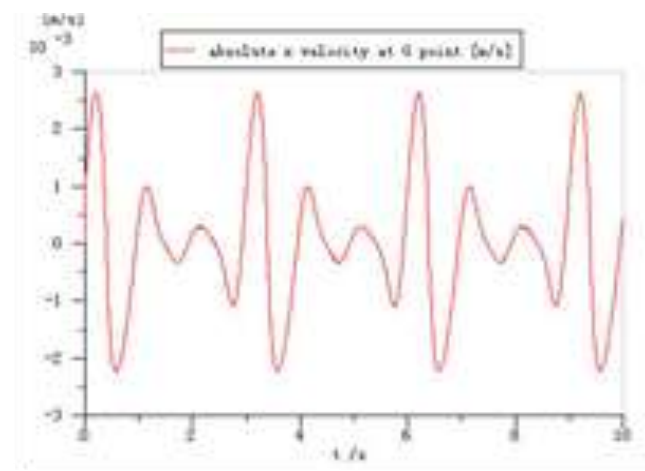

Figure 6: Absolute $x$ velocity at $G$ point

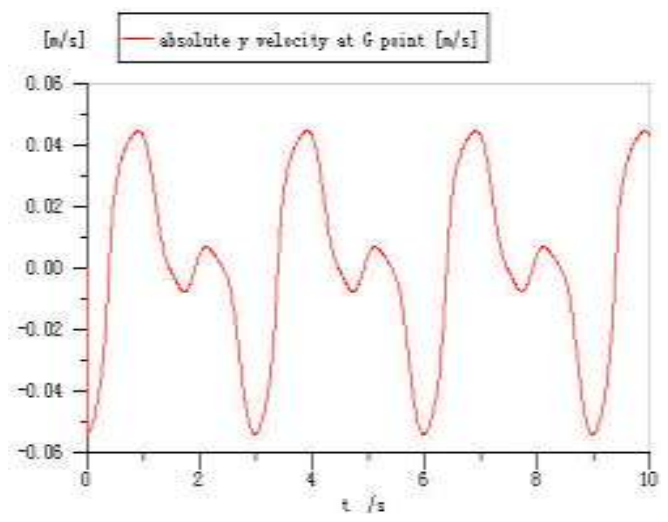

Figure 7: Absolute y velocity at G point

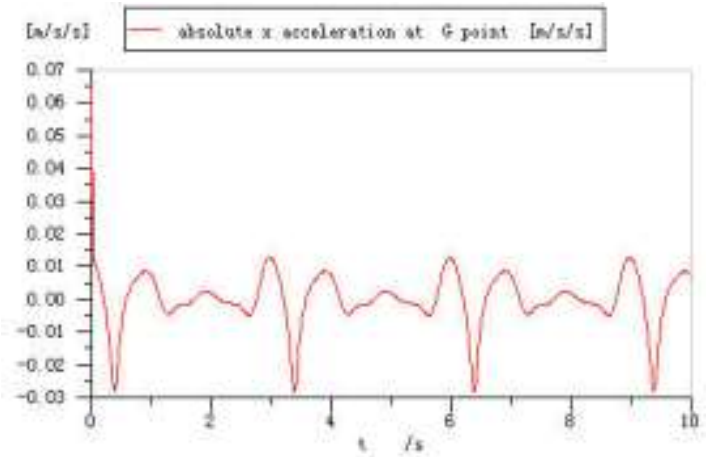

Figure 8: Absolute $x$ acceleration at $G$ point

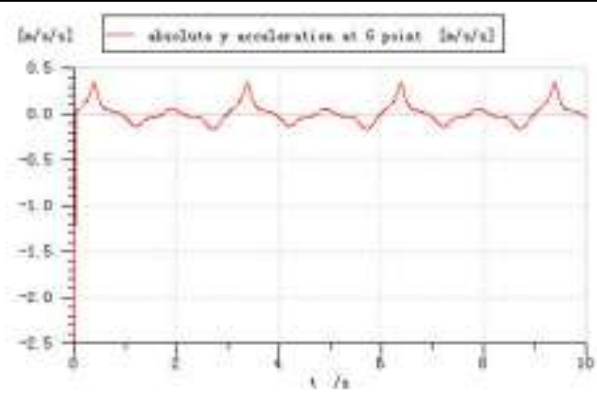

Figure 9: Absolute y acceleration at G point

A new document was built in SimMultibody and then sketch mode was entered. The related components in SimMultibody were selected to build linkage. Only the connection relation between each member and articulated point is needed to be determined in draft mode, without geometry size and position relationship being considered. The articulated point A was simulated by an element. Torsion spring model and angularvelocity input element in SimMultibody were successively added on the element. Thus, an input of constant angular velocity was formed. The built model is shown in Figure 2, and animation screenshot in Figure 3. The position, velocity and acceleration of G are shown in Figures4-9.

\section{CONCLUSIONS}

The kinematics simulation analysis of plane ten-bar mechanism of transmission latch needle of warp knitting machine was performed on the basis of SimMultibody. The results and curve corresponding to mechanism are obtained, which provides a basis for further research in future.

The use of SimMultibody to conduct the modeling and simulation of mechanism system can avoid building complex mathematical model. It only needs to select corresponding modules to build a physical model of mechanism and set relevant parameters according to the connection relation among mechanism components. Thus, the motion response law of mechanism can be obtained, and Virtual Reality Toolbox to perform realtime animation demonstration of mechanism motion. In a word, it has many advantages such as convenient and intuitive system modeling, powerful simulation function and automatic modeling and analysis.

SimMultibody was applied to the design and analysis of complex multi-bar mechanism in textile machinery. Studies have shown that various parameters of mechanism can be obtained by doing this, and a scientific basis is provided for product design. Moreover, product development efficiency can be greatly improved, thus saving costs and time and improving efficiency.

\section{REFERENCES}

[1] Qi, Z., Gaoming, J., Fenglin, X. 2013. Warp knitting machine CNC system key technology and its development. Textile Leader, 4, 77-80.

[2] Fenglin, X. 2010. New warp-knitting machinery technology and development tendency. China Textile Leader, 9, 55-56.

[3] Jianjun, M. 2008. Fuzzy comprehensive assessment of cam moving rule in high speed warp knitting machine. Journal of Textile Research, 29 (12), 101-103.

[4] Zheng, D., Zhou, L., Liang, D. 2013. Modeling and Simulation of Kinematics for Planar R-RRR-RRP Mechanism Based on Assur Rod Group Theory. Applied Mechanics and Materials, 423-426, 1879-1883.

[5] Danian, H., Zhihong, H. 2012. Linkage design and application innovation. Beijing: China Machine Press.

[6] Dang, Zheng, X., Zhou, L.S., Liang, D. 2013. Dynamic Performance Analysis and Simulation of Hybrid-Driven Seven-Bar Mechanical Press with Double Cranks. Advanced Materials Research, 834-836, 1327-1332. 